$30(1.6 \%)$ occasions. In the light of this experience the routine employment of doctors may be unnecessary and involve an uneconomical use of special skills.

Despite the shortage of doctors in many countries much can be done by the organization of available resources to bring earlier skilled attention to patients with acute coronary heart disease. We believe that the routine use of paramedical personnel in cardiac resuscitation within the limits of economic circumstances is the best system for most urban areas using a hospital rota system. Nevertheless, we envisage a further improvement in the efficiency of the service when radio-telemetry is incorporated to provide immediate and continuous transmission of E.C.G. information ${ }^{7}$ about the patient before admission, thus allowing the doctor on rota duty to advise the family doctor and ambulance crew about immediate management. Improved public and professional education should see a reduction in the present unsatisfactory time interval between the onset of symptoms and the arrival of the ambulance.

The resuscitation of a limited number of patients with primary ventricular fibrillation is an important benefit of mobile coronary care. Other benefits, including education of medical and paramedical personnel in cardiopulmonary resuscita- tion and in the recognition and management of acute coronary heart disease, may prove to be important long-term advantages of the service.

The Irish Heart Foundation wishes to acknowledge with thanks the co-operation of the Dublin Health Authority; the Dublin Fire Brigade Ambulance crews; the governors and medical staffs of the Dublin Voluntary Hospitals; and the doctors and nurses attached to the coronary care units. Special thanks must be extended to $\mathrm{Mr}$. Noel Gleeson, Director of the Stillorgan Ambulance Service, for the major part he has played in the success of this mobile coronary care programme, and to his crew members, who have worked on the programme with ability and enthusiasm.

\section{References}

1 Lawrie, D. M., et al., Lancet, 1968, 2, 523.

2 Adgey, J. A. A., et al., Lancet, 1969, 1, 1169.

3 Dewar, H. A., McCollum, J. P. K., and Floyd, M., British Medical fournal, $1969,4,226$.

4 McNeilly, R. H., and Pemberton, J., British Medical fournal, 1968, 3, 139. 5 Pantridge, J. F., and Geddes, J. S., Lancet, 1966, 1, 807.

- World Health Örganization, Role of Mobile Coronary Care Units, Copenhagen, W. H.O. 1970 .

? Uhley, H. N., American Heart fournal, 1970, 80, 838.

\title{
Computers in Medicine
}

\section{Doctors as Decision-makers : A Computer-assisted Study of Diagnosis as a Cognitive Skill}

\author{
T. R. TAYLOR, J. AITCHISON, E. M. MCGIRR
}

British Medical fournal, 1971, 3, 35-40

\section{Introduction}

In recent years considerable effort has been directed towards the construction of computer programmes to aid diagnosis in specific areas of medicine. Examples are reported by Warner et al. ${ }^{1}$ for congenital heart disease; by Overall and Williams, ${ }^{2-7}$ Overall and Hollister, ${ }^{8}$ Boyle et al.,9 Fitzgerald et al., ${ }^{10}$ and Taylor $^{11}$ for thyroid disease; by Bishop and Warner ${ }^{12}$ for polycythaemia; by Lodwick et al. ${ }^{13} 14$ for bone tumours; and by Ferriss et al. ${ }^{15}$ for Conn's syndrome-see also Taylor. ${ }^{16} \mathrm{~A}$ necessary step towards the acceptance by clinicians of the value of such computer-aided diagnosis is the comparative study of the diagnostic behaviour of clinicians with the diagnostic performance of the computer programme. Such comparisons are usually based on rather crude differences between the results obtained by the clinician and by the computer. For example, the difference in their percentages of correct diagnoses or in the average probability ratings they assign to the correct diagnosis has been commonly used.191217 Moreover, in such studies diagnosis has been considered only at the end of the complete process of data collection.

Many writers ${ }^{18-20}$ have pointed out that the diagnostic process is essentially a sequence of decisions, and some ${ }^{21}$ have suggested that it would be interesting to compare clinician and computer in the context of such a sequential decision-making process.

The primary purpose of this paper is to explain how such a sequential comparison may be made between clinician and computer. The objective techniques developed are illustrated
University Department of Medicine, Royal Infirmary, Glasgow, $\mathbf{C} .4$ T. R. TAYLOR, M.R.C.P.(ED., GLASG.), D.P.M., Lecturer in Medicine

E. M. MCGIRR, M.D., F.R.C.P.(LOND., ED., GLASG.), Muirhead Professor of Medicine

University of Glasgow, Department of Statistics

J. AITCHISON, M.A., F.R.C.S. D., Titular Professor of Statistics 
by results from a study of six clinicians tackling identical problems in the simple three-disease system of non-toxic goitre. In the course of this study the possible importance of personality factors in influencing diagnostic behaviour became apparent. Moreover, while the techniques of comparison have been primarily devised for the analysis in depth of experienced clinicians they are easily adapted to the teaching of some of the skills underlying diagnosis in a simple yet illuminating way. These aspects of personality and teaching form important subsidiary considerations.

At each cycle of the diagnostic process (Table I) the following factors must be weighed up: (1) the advantages of making an

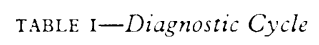

immediate correct diagnosis; (2) the consequences of making an overhasty misdiagnosis; and (3) the financial cost, discomfort, inconvenience, and delay due to further diagnostic investigation before treatment is begun.

While early work 22 on sequential diagnostic procedures attempted to take some account of costs the actual role of financial costs in diagnostic decision-making within the National Health Service is certainly not clear. This role would obviously differ from that in other countries where clinicians are of necessity more cost-conscious. Certainly the clinicians in the present study, when attempting to estimate the costs of the laboratory tests used, confirmed their own impressions of how unfamiliar they were with thinking in such financial terms. In the design of this study, therefore, we have tried to eliminate such cost-consciousness that may exist by constantly reminding the participants during each case that tests were to be regarded as equally available and cost-free. Our approach has therefore been to adopt a more recent type of computer-assisted decisionmaking which makes use of a basic measurement of information. ${ }^{11}$ At each cycle in the diagnostic sequence the procedure selects the test which promises to be the most informative in such a way that a great reduction in the number of tests performed on a patient may be possible.

\section{Materials and Method}

Altogether 20 cases of non-toxic goitre were used-16 different cases, of which 4 were repeated unknown to the participants. The cases were presented to each of six clinicians-members of the staff of the thyroid clinic of Glasgow Royal Infirmaryin two sessions of 10 cases. The cases were 2 of Hashimoto's disease, 12 (4 repeated) of simple goitre, and 2 of thyroid carcinoma; this distribution was chosen as reasonably representative of the frequencies $(10,89$, and $1 "$.$) with which these$ diseases are seen in the clinic. The cases were selected to provide variation in difficulty by the objective method of classification shown in Taylor's Fig. 4. ${ }^{11}$ This method groups cases according to the number of tests required to reach a diagnostic probability level of 0.99 by the procedure contained in the computer programme. The cases were presented to each clinician in an identical random order so that no clear pattern of diagnosis or ease of diagnosis was apparent.

The cases were presented in the form of abstracted case records with the results of 30 tests (Table II) available on each. Each clinician used a recording sheet (Fig. 1). He began by recording his assessment of the previous probabilities (incidence rates) of the three diseases at the clinic. He then selected his first test, was told the outcome, and entered his revised assessment of the probabilities of the three diseases in the light of this information. He noted this by dividing the strip (of unit length in Fig. 1) into three parts, and also recorded the probabilities
TABLE II-Tests used in Study

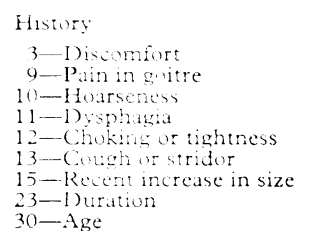

Examination

6--Fixation to tissues

7-Cervical lymph glands

8-Pramidal lobe

2.4-Istimated size of gland

25 -Consistency

Special Examination

4-Tracheal deviation (or compression on $x$-ray film) 5-Laryngeal palsy (indirect laryngoscopy)

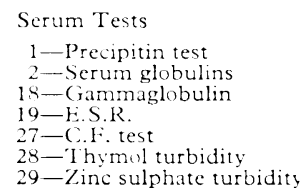

29-Zinc sulphate turbidity
Special Tests $14-\mathrm{KClO}_{4}$ discharge 17-Butamol Extractable 101 $20-24$-hour 22 - 48 hours P B ${ }^{131}$

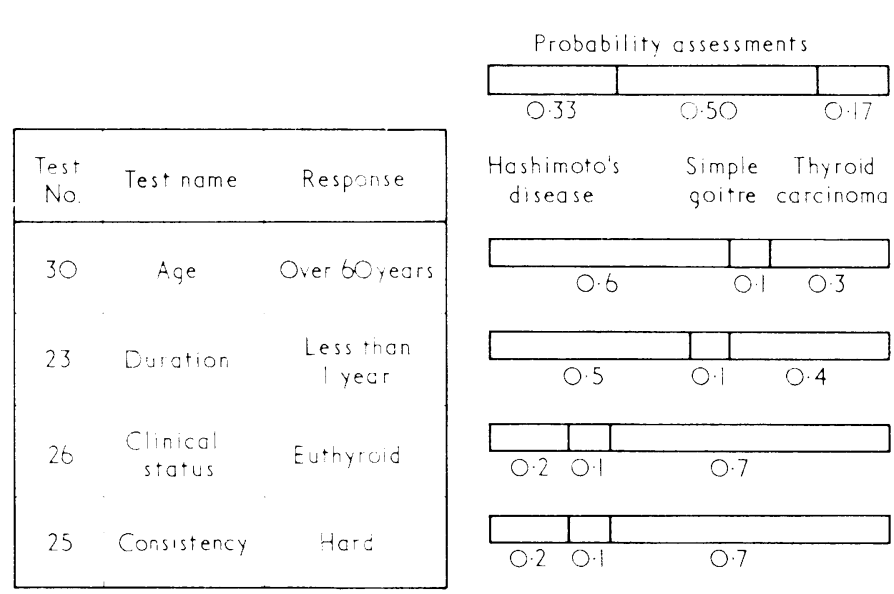

FIG. 1-Recording sheet used in study. Example shown is of the first four tests of Clinician 3 diagnosing the case of thyroid carcinoma of Fig. 3.

below. He then selected his next test, was told the outcome, and again altered the probabilities as he thought fit. He continued in this way till he was satisficd that he had sufficient information to make a diagnosis.

He was allowed to select tests in any sequence and to "back track"-for example, from a laboratory test to an item of history. He was repeatedly reminded that all tests were equally available and that costs should not be taken into account.

\section{Analysis}

The principles underlying the analysis depend on statistical ideas of inference and decision theory and of information theory, particularly the work of Shannon, 23 Kullback and Leibler, ${ }^{24}$ and Lindley. ${ }^{25}$ While it is undesirable to give a detailed technical account of these in this paper an attempt is made to convey the ideas at an intuitive level. The reader interested in elementary background reading is referred to Lusted, ${ }^{21}$ Raiffa, ${ }^{26}$ and Aitchison. ${ }^{27}$

The computer programme used here ${ }^{11}$ operates on the following assumptions. (1) For a given disease the tests are statistically independent. (2) The tests are equally available without cost. (3) The test selected at each diagnostic cycle (Table I) is that which, relative to the position attained, promises to provide most additional information by the end of the cycle. (4) When the result of a test is known the probabilities of the diseases are updated by the use of Bayes's theorem (see Boyle et $a l^{9}$ and Lusted ${ }^{21}$ for uses of this approach in medical situations). 
A clinician's decision-making behaviour at each diagnostic cycle is indicated by (1) his choice of test and (2) his updating of the disease probabilities after learning the outcome of the test.

At the start of each cycle the clinician is uncertain about the diagnosis. The degree of his uncertainty is indicated by the current probabilities he is quoting and can indeed be quantified. For example, the probabilities $(1 / 3,1 / 3,1 / 3)$ assigned to Hashimoto's disease, simple goitre, and thyroid carcinoma correspond to a maximum degree of uncertainty, since the clinician is equally torn between the three possible diagnoses. At the opposite extreme, in such a probability assessment as $1,0,0$, the clinician is stating that the diagnosis is definitely Hashimoto's disease, and there is no uncertainty in this view. Such an assessment as $0 \cdot 5,0 \cdot 3,0 \cdot 2$ clearly lies intermediate to these two extremes in its associated degree of uncertainty. This intuitive ordering is reflected in the quantified degrees of uncertainty $U$ calculated as $1.58,0$, and 1.48 bits (standard units for measuring uncertainty and information) for the probability assignments $1 / 3,1 / 3,1 / 3 ; 1,0,0$; and $0.5,0.3,0.2$ respectively.

An illuminating method of presenting a clinician's path to a diagnosis for this three-disease system is to use an equilateral triangle, HSC (Fig. 2 (i)), whose vertices represent the three diseases-Hashimoto's disease, simple goitre, thyroid carcinoma.
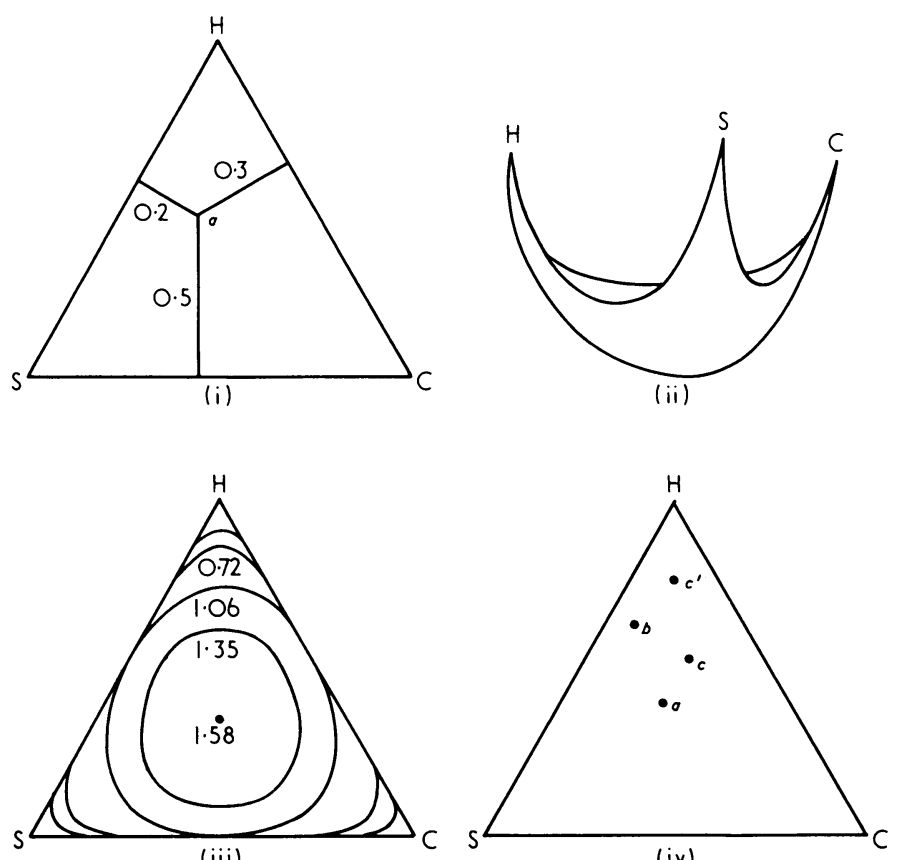
FIg. 2-(i) Representation of a probability assessment in an equilateral
triangle. (ii) Triangular bowl of uncertainty. (iii) Contours of uncertainty. triangle. (ii) Triangular bowl of uncertainty. (iii) Contours of uncertainty. (iv) Examples of conservative and liberal use of data.
disease. $\mathrm{S}=$ Simple goitre. $\mathrm{C}=$ Thyroid carcinoma.

If the altitude of this triangle is of unit length then each point within the triangle can be used to represent a probability assessment for the three diseases. In Fig. 2 (i) the distance of the point from the side opposite a disease vertex is the probability placed on that disease-for example, the distances of $a$ from the sides $\mathrm{SC}, \mathrm{CH}$, and $\mathrm{HS}$ are $0.5,0.3,0.2$, therefore $a$ represents a probability assessment of $0.5,0.3,0.2$ on Hashimoto's disease $(\mathrm{H})$, simple goitre $(\mathrm{S})$, and thyroid carcinoma $(\mathrm{C})$. Each time the clinician changes his probability assessment he moves from one point to another and so traces out a diagnostic path within the triangle; his movement towards a vertex (for example, $\mathrm{H}$ ) represents his changing proximity to that diagnosis (Hashimoto's disease).

We can provide an extra dimension to this visual picture by associating with each point in the triangle its degree of uncertainty discussed above. If each degree of uncertainty is represented by depth below the surface of the triangle we trace out as the uncertainty surface a kind of triangular bowl (Fig. 2 (ii) ). This surface reaches to the level of the triangle only at the three vertices. The uncertainty contours ${ }^{28}$ are shown in Fig. 2 (iii) in the same way as a contour map shows ocean depth. As he collects information the clinician moves around the bowl attempting to climb towards one of the vertices and so to arrive at a firm diagnosis.

The widely different diagnostic paths taken by Clinicians 3,5 , and 6 when dealing with the same case of thyroid carcinoma are shown in Fig. 3; also shown by a broken line is the path taken by the computer programme. For each path the

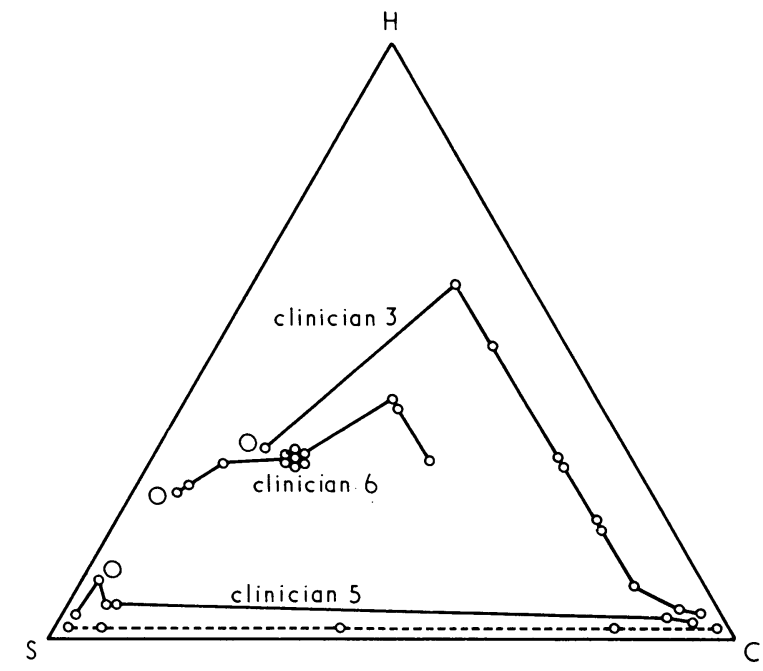

FIG. 3-Diagnostic paths of three clinicians (Nos. 3, 5, and 6 ) and of the computer programme (broken line) for a case of thyroid carcinoma. $\mathrm{O}=$ Starting position.

point labelled $\mathrm{O}$ is the starting position. For a clinician this starting point corresponds to his view of the incidence rate of the three diseases in the clinic; for the computer programme this starting point corresponds to the observed incidence rates in the thyroid clinic-namely, $0 \cdot 10,0.89$, and 0.01 for Hashimoto's disease, simple goitre, and thyroid carcinoma. The successive points marked along a route show the positions moved to after successive tests. The serial numbers of the tests in the order selected were: Clinician $3-30,23,26,25,16,13$, 7,1 , and 10; Clinician 5-15, 23, 7, and 25; Clinician 6-15, 30, $23,3,10,12,11,25,16,6,7$, and 1 ; and the computer programme-1, 9, 28, 25, 30, and 7 . The cluster of points on the diagnostic path of Clinician 6 corresponds to his position after tests $23,3,10,12,11,25$, and 16 ; in other words, having arrived there after test 23 he made no alteration to his probability assessment for the next six tests he selected. In each path the final point shown is that at which the diagnosis of thyroid carcinoma was correctly made in each case.

The plotting of such paths clearly indicates a picture of broad variation between clinicians and between some clinicians and the computer programme. We can obtain, however, a more penetrating analysis by forcing a comparison between clinician and computer in each diagnostic cycle. To allow a fair comparison the computer programme is made to operate with the clinician's probabilities at the start of each cycle. In each such cycle there are then two ways in which differences between the clinician's behaviour and the computer programme may arise. (1) The test chosen by the clinician may differ from the computer selection of test. (2) The updating of the probabilities as stated by the clinician may differ from the updating made by the computer. A diagrammatic representation of the cycle and the possible points of discrepancy are given in Fig. 4. At each cycle and for each clinician four measures may be computed to assess various aspects of these differences. The theoretical basis 


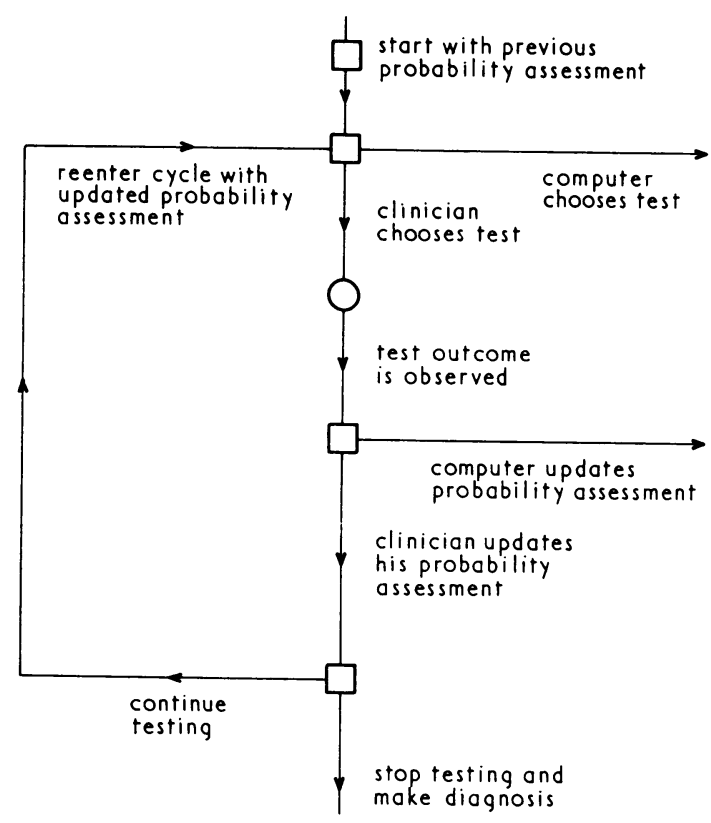

FIG. 4-Flow diagram of clinician's diagnostic cycle showing possible sources of discrepancy with computer programme.

of these measures, here described only briefly, is discussed in detail by Aitchison and Taylor. ${ }^{29}$

\section{Measures of Discrepancy}

Test-selection Discrepancy, T.-At a particular stage of the diagnostic process the value of any test can be measured in terms of the amount of information it then provides-that is, the amount of uncertainty it removes. At any stage of diagnosis it is therefore natural to select the test which promises, or is expected, to provide most additional information to discriminate between the three diseases. The computer programme does this (assumption iii). The amount by which this maximum expected gain of information exceeds the corresponding gain associated with the test chosen by the clinician is termed the test-selection discrepancy $T$.

Inference Discrepancy, I.-A measure of the discrepancy between the clinician's interpretation of a test outcome and the computer's interpretation must be some overall measure of the differences between the two sets of updated probabilities. One appropriate measure is the Kullback and Leibler ${ }^{24}$ measure of discrimination, which we denote by $I$. This reads zero when the sets of updated probabilities coincide-that is, when there is agreement between the clinician and the computer programmeand positive when there is any disagreement. The greater the disagreement the greater the value of $I$.

Conservativism-Liberalism Index, L.-In Fig. 2 (iv), starting from the attained position $a$, suppose that the computer move goes to $b$ while the clinician's move takes him to $c$. The movement from $a$ to $c$ represents a smaller reduction in uncertainty than the move from $a$ to $b$. The clinician may therefore be said to be acting conservatively in his use of data relative to the computer programme in this case. If, on the other hand, he moves to $c^{\prime}$, a position of less uncertainty than $b$, he is over-using the data or acting liberally relative to the computer programme. For other configurations of $a, b, c$ similar arguments apply. A measure of this type of discrepancy is the difference, $L$, in the degrees of uncertainty associated with $b$ and $c$ taken positively for liberal and negatively for conservative behaviour. For a fuller discussion see Aitchison and Taylor. ${ }^{29}$

Headway Towards Correct Diagnosis, D.-This is a simple measure to show, separately for each cycle, the relative progress of clinician and computer programme towards the correct diagnosis. It is simply calculated as the difference between the updated probabilities assigned to the known correct disease taken positively if the clinician's assessment is the larger.

\section{Illustrative Examples}

The absolute differences between clinicians and the computer programme are shown in Fig. 3. The corresponding cycle-bycycle discrepancies are illustrated in Fig. 5, which gives the (U, T, I, L, D) profile for each clinician in the same case of thyroid carcinoma. The effectiveness of this form of analysis is shown by some observations on these results. We emphasize that no value judgements are implied in these comparisons. The

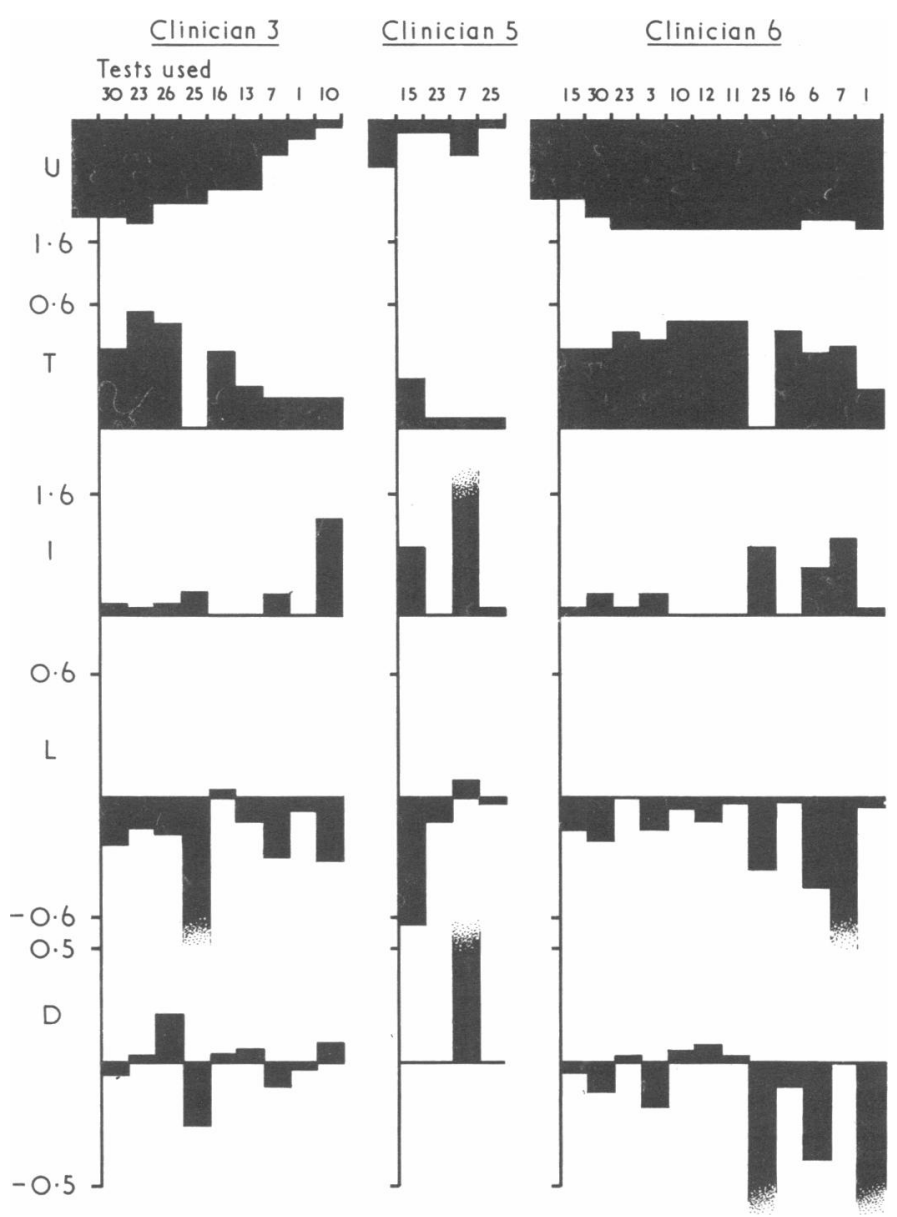

FIG. 5-Profile analysis of three clinicians for the case of thyroid carcinoma of Fig. 3. $U=$ Degree of uncertainty after test. $T=$ Test discrepancy. $\mathrm{I}=$ Inference discrepancy. $\mathrm{L}=$ Liberalism index. $\mathrm{D}=$ Headway towards diagnosis.

computer programme merely provides us with an objective indirect comparison among a number of clinicians.

Clinician 3 shows a large discrepancy in test selection $(T)$ but is close to the computer programme in interpreting these tests (I). He is generally conservative in his use of data $(\mathrm{L}<\mathrm{O})$ but is mildly liberal in the use of information from test 16 .

Clinician 5 reduces the degree of uncertainty (U) more rapidly than the others. He is also much nearer the computer programme in test selection $(T)$ and uses fewer tests than Clinicians 3 and 6 to reach a correct diagnosis. In test 7 he is liberal $(L>0)$ in probability estimation and is ahead of the normative model in progress towards the diagnosis (D).

Clinician 6 is more uncertain at the end of his case than when he began $(U)$; he is consistently conservative $(L<0)$ and shows a large discrepancy in test selection ( $T)$. His inference discrepancy (I) is low, but this may be explained by the fact that 
the tests he selects provide little information when this is calculated in accordance with the computer programme.

The three clinicians select test 25 at different stages of their diagnostic paths, and yet the test-selection discrepancy $T$ is zero for each. This is simply explained by test 25 being highly informative for most starting positions in the triangle.

\section{Discussion}

The choice of thyroid diseases for our study is in part due to the availability of case material, but also because there are many excellent and sophisticated investigative procedures available to provide a firm diagnosis in most cases. It is therefore a suitable system within which to develop decision-making techniques with a reasonable hope of short-term success. In other systems-for example, the central nervous system, liver disease, or alimentary disease-the problems of diagnosis and treatment are much more complex. It is hoped, however, that techniques developed within the thyroid field may be extended to other areas at a later date. ${ }^{30}$ This particular study is an important part of an interrelated series of investigations of computer-assisted decision-making in thyroid disease being pursued at present in the department of medicine in the Royal Infirmary, Glasgow. ${ }^{31}$

Previous comparisons of actual decision-making behaviour with a normative procedure (see, for example, Phillips and Edwards ${ }^{32}$ ) have concentrated on the testing of individuals as probability estimators in experimental game-playing situations where small monetary rewards are paid for correct decisions. The present study with three diseases has the advantage of retaining the simplicity of these artificial settings while being accepted as reasonably realistic by the clinicians taking part. Whether or not the assumptions of the present programme are accepted it is certainly useful in accentuating the considerable variations in approach by different clinicians.

The profiles in Fig. 5 are representative of the range of differences we have found throughout this study. Since some of these variations may be accounted for by personality factors, the participating clinicians (much to their credit) were persuaded to complete two personality profiles, the Eysenck personality inventory ${ }^{33}$ and the 16 p.f. test. ${ }^{34}$ These are aimed at giving an overall view of personality traits and were assessed independently by a clinical psychologist. Some preliminary results, indicating some relationship between obsessionalism, accuracy of diagnosis, and the number of tests used, are given in Table III.

TABLE III-Preliminary Analysis of Performance of Six Clinicians in the Study. Clinicians are Ranked in Decreasing Order of Obsessionalism

\begin{tabular}{|c|c|c|c|c|c|c|}
\hline & \multicolumn{6}{|c|}{ Clinicians } \\
\hline & 1 & 6 & 5 & 4 & 2 & 3 \\
\hline $\begin{array}{l}\text { No. of correct diagnoses out of } 20^{*} \\
\text { No. of tests used } \ldots\end{array}$ & $\begin{array}{r}20 \\
213\end{array}$ & $\begin{array}{r}20 \\
174\end{array}$ & $\begin{array}{r}19 \\
141\end{array}$ & $\begin{array}{r}18 \\
141\end{array}$ & 18 & $\begin{array}{r}17 \\
153\end{array}$ \\
\hline
\end{tabular}

* Computer 18/20.

Previous studies mentioned have shown that subjects in gameplaying situations are always conservative. ${ }^{32}$ In the case shown in Fig. 5 liberalism occurred in some tests. If liberalism persists in some clinicians (when reasonable account is taken of costs and of the interdependence of tests) this may suggest that these individuals are using some form of pattern recognition. The relationship between conservativism and obsessionalism and the question whether, for example, obsessional personalities make decisions (diagnoses) impulsively will also be the subjects of further studies. If any association can be shown between diagnostic skill and such personality or cognitive factors it may be possible to teach individuals to develop such factors that are valuable and to avoid those which are a hindrance-for example, impulsiveness.

The computer programme with its assumption of the independence of tests, its limited outlook to the end of the current cycle in its choice of test, and the absence of costs, is a very simple-minded one. The independence assumption is at the heart of almost all previous work in this field. ${ }^{21}$ It is adopted here simply because insufficient data are available. The prospective survey at present being conducted in the thyroid clinic of the Royal Infirmary, Glasgow, should provide sufficient data on two-test dependence, and we shall then adopt a programme incorporating this dependence and looking two cycles ahead in its test selection. Aitchison and Taylor ${ }^{29}$ provide a fuller discussion of the effects of these assumptions and of studies in artificial situations with the more sophisticated programme.

A work study and a cost benefit analysis are being conducted simultaneously with the prospective survey and will allow financial costs to be considered later. It is also simple to impose a grouping of tests into some hierarchical scheme of historytaking, physical examination, and laboratory tests to meet certain practical restrictions placed on the system by clinic organization.

While the visual appeal of the simple triangle and bowl of uncertainty (Fig. 2 (ii)) as an expository aid is restricted to a three-disease system, it should be emphasized that for a system with more diseases and tests the profile analysis does not alter in form. It is this fact that has stimulated an investigation of the profile analysis as a possible teaching aid.

\section{Teaching of Clinical Decision-making Skills}

When the decision-making which underlies diagnosis is viewed as a cognitive skill then the importance of studies such as this one for medical teaching becomes clear. With the methods of analysis described above it should be possible to teach some of the skills required to deploy effectively a group of tests-for example, in thyroid disease. Clinicians in the study have already said that they have been forced to think more critically about their approach to diagnostic problems. An important development in teaching such interpretative skills is to make the profile analysis immediately available during the diagnosis of a case. A clinician (whether undergraduate or postgraduate), but observing the effects on his profile (Fig. 5) of a particular choice of test or of his updating of probabilities, learns to assess the discriminating power of clinical information. Clearly when

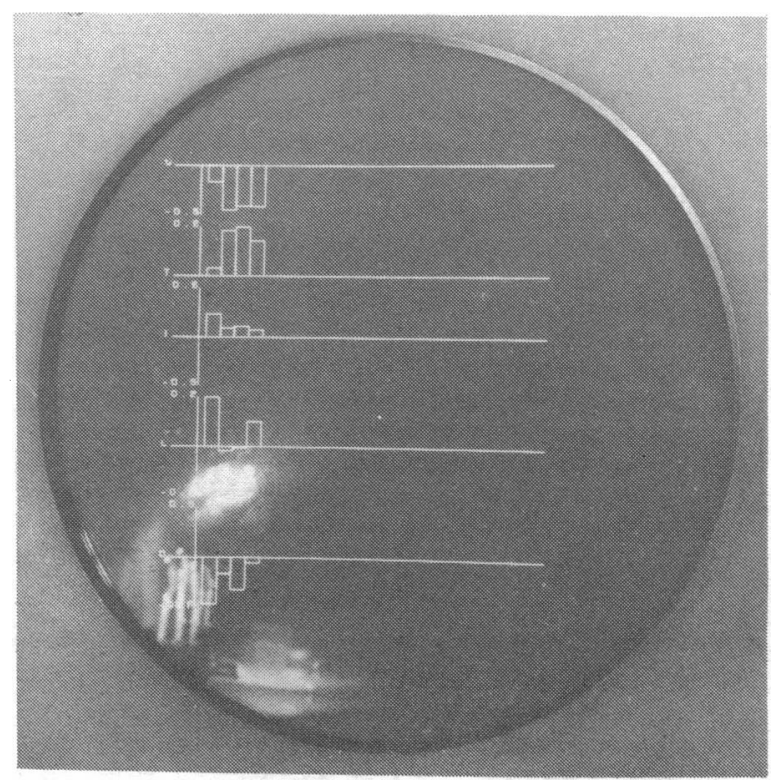

FIG. 6-Instantaneous profile analysis as presented on interactive visual display. 
costs are taken into account comparisons between clinicians will become more complex. By a deliberate attempt to minimize the effect of costs in this initial study we have sought to highlight the information-processing aspects of diagnosis. The programmes developed for the analysis used in this study have been extended to provide a rapid, on-line interactive analysis of decision-making with a teletype.

A much more sophisticated interactive terminal with a television-type visual display and a light-pen facility (PDP 338) has now been adapted to present the analysis almost instantaneously (Fig. 6). This display is controlled by a PDP 8 computer linked to a time-sharing ICL KDF9 computer. A case record is selected from the computer store. At each diagnostic cycle the clinician indicates his choice of test (from a list displayed on the consol tube) and his subsequent updating of the probabilities (by dividing a unit strip on the face of the consol tube) by the use of the light-pen. After each cycle the profile analysis for that cycle is displayed within a few seconds (Fig. 6). If the clinician or student is choosing his test and updating his probabilities on the principles underlying the computer programme no rectangle depicting discrepancy will appear. The nature of any discrepancy can be quickly ascertained from these rectangles. A facility to back track to reselect a test and reassess his probabilities is provided, so allowing the user to learn by this analysis of his performance.

Just as important as its value as a teaching system will be the use of such an interactive terminal to study clinicians in greater detail when tackling a wide variety of cases and to assess the effect of the introduction of costs on clinicians' decisions. As information about decision-making is acquired it can be used to "update" the computer programme, making it more realistic and so more effective for teaching.

\section{Conclusions}

Medical diagnosis can be viewed as problems in sequential decision-making, and statistical decision theory can provide a useful framework within which to investigate these skills.

The study described here illustrates the power and flexibility of such a framework. The differences between clinicians suggest that it may well be impossible to produce a model to simulate all doctors. There is a prima-facie case for studying the influence of personality factors on strategies of diagnosis. A computer programme has been developed to provide rapid detailed analysis of each stage or cycle in diagnosis. This has been extended to use either an on-line computer link or a visual display with light-pen facility to study clinical decision-making in great detail. The scope of such an approach gives some hope of yielding not only an objective computerized aid to diagnosis but also a framework within which to provide training in diagnostic skills.
We wish to record our grateful thanks to the anonymous clinicians of the department of medicine of the Royal Infirmary, Glasgow, who so willingly and enthusiastically collaborated in this study, and to Miss Mary Nicolson, S.R.N., our programmer, for her considerable contribution to the success of the study. This work was supported in part by a grant from the Medical Research Council.

\section{References}

1 Warner, H. R., Toronto, A. F., Veasey, L. G., and Stephenson, R., Fournal of the American Medical Association, 1961, 177, 177.

Overall, J. E., and Williams, C. M., Behavioral Science, 1961, 6, 134.

Overall, J. E., and Williams, C. M., Medizinische Dokumentation, 1961, 5, 51.

4 Overall, J. E., and Williams, C. M., Medizinische Dokumentation, 1961, 5, 78.

5 Overall, J. E., and Williams, C. M., fournal of the American Medical Association, 1963, 183, 307.

6 Overall, J. E., and Williams, C. M., in Proceedings of Third San Diego Symposium for Biomedical Engineering, ed. Fogel, L. J., and George, F. W., Symposium for Biomedical Engineering, ed.

Overall, J. E., and Williams, C. M., in Data Acquisition and Processing in Biology and Medicine, ed. K. Enstein. New York, Pergamon, 1964.

${ }^{8}$ Overall, J. E., Hollister, K. and Hollister, L. E., fournal of The American Medical Association, 1964, 187, 583.

${ }^{9}$ Boyle, J. A., et al., Quarterly fournal of Medicine, 1966, 35, 565.

Fitzgerald, L. T., Overall, J. E., and Williams, C. M., American fournal of Roentgenology, Radium Therapy, and Nuclear Medicine, 1966, 97, 901.

11 Taylor, T. R., fournal of the Royal College of Physicians of London, 1970, 4,188 .

12 Bishop, C. R., and Warner, H. R., Computers and Biomedical Research, $1969,2,486$

${ }_{13}$ Lodwick, G. S., Investigative Radiology, 1966, 1, 72.

14 Lodwick, G. S., Turner, A. H., jun., Lusted, L. B., and Templeton, A. W., Fournal of Chronic Diseases, 1966, 19, 485.

15 Ferris, J. B., et al., Lancet, 1970, 2, 995.

16 Taylor, T. R., Principles of Medical Computing, p. 82. Oxford, Blackwell, 1967.

${ }^{17}$ Crooks, J., Murray, I. P. C., and Wayne, E. J., Quarterly fournal of Medicine, 1959, 28, 211.

${ }^{18}$ Ledley, R. S., Use of Computers in Medicine and Biology. New York,

Wiley, 1966.
19 Ledley, R. S., and Lusted, L. B., Science, 1959, 130, 9.

${ }^{2}$ Hamilton, M., Clinicians and Decisions. Leeds, Leeds University Press, 1966.

${ }^{21}$ Lusted, L. B., Introduction to Medical Decision Making. Springfield, Illinois, Thomas, 1968.

22 Gorry, G. A., and Barnett, G. O., Computers and Biomedical Research, $1968,1,490$.

${ }^{23}$ Shannon, C. E., Bell System Technical fournal, 1948, 27, 379.

24 Kullback, S., and Leibler, R. A., Annals of Mathematical Statistics, 1951,

22, 79.
${ }^{25}$ Lindley, D. V., Annals of Mathematical Statistics, 1956, 27, 986. ${ }_{25}^{25}$ Lindley, D. V., Annals of Mathematical Statistics, 1956, 27, 986.

${ }^{27}$ Aitchison, J., Choice Against Chance: An Introduction to Statistical Decision Theory. Reading, Massachusetts, Addison-Wesley, 1970.

${ }^{28}$ Schmidt, S. A., Measuring Uncertainty: An Elementary Introduction to Bayesian Statistics. Reading, Massachussetts, Addison-Wesley, 1968.

${ }^{29}$ Aitchison, J., and Taylor, T. R., in preparation, 1971.

${ }^{30}$ Taylor, T. R., Science fournal, 1970, 6, 81.

31 Taylor, T. R., Contribution to discussion of Aitchison, J., fournal of the Royal Statistical Society, 1970, A 133, 206

32 Phillips, L., and Edwards, W., Fournal of Experimental Psychology, 1966,

72, 346.
${ }^{33}$ Eysenck, H. J., and Eysenck, S. B. G., Eysenck Personality Inventory (Form B). London, University of London Press, 1965

${ }^{34}$ Cattell, R. B., and Eber, H. W. (1963). Sixteen Personality Factor Questionnaire (Form B). Institute for Personality and Ability Testing, Illinois. 